\title{
Zur Theaterpädagogik in der Bundesrepublik Deutschland
}

\author{
Ein Überblick
}

\section{Gerd Koch}

\begin{abstract}
Zusammenfassung
Der folgende Text war die Grundlage für einen Vortrag, den ich am 5. April 2008 in der Staatsbibliothek in Ankara hielt - vor einem vorzüglich interessierten Publikum, dem ich herzlich danke! Die akademischen Titel im Beitrag werden bewusst genannt, um anzuzeigen, dass Theaterpädagogik durchaus kompetent mitredet im Konzert universitärer Fächer. Kultur- und Bildungspolitik ist in Deutschland Sache der einzelnen Bundesländer. Das wirkt sich auch auf die mit dem Lernort Schule verbundene Theaterpädagogik aus. Ich werde im Folgenden einen Überblick über das Gesamtgeschehen und über die neuere Geschichte der Theaterpädagogik in Deutschland geben. Dazu werde ich beispielhaft und egoistisch (und das heißt auch: ungerecht) verfahren. Ich werde die Entwicklung der „Zeitschrift für Theaterpädagogik“ zur Richtschnur nehmen.
\end{abstract}

\section{Lehrstück ... Theater ... Pädagogik ...}

Die Zeitschrift für Theaterpädagogik gibt es erst seit 2005 unter dem Namen „Zeitschrift für Theaterpädagogik“. Gegründet wurde sie jedoch bereits 1985, damals aber unter dem Titel „Korrespondenzen“ und mit diesem Untertitel ” ... Lehrstück ... Theater ... Pädagogik ..... Die drei Begriffe des Untertitels wurden getrennt von einander geschrieben. Also: Lehrstück, Theater, Pädagogik. Sie wurden zusätzlich noch durch drei Punkte voneinander getrennt. Ja, die Unterzeile des Titels begann selbst mit drei Punkten - wie, um eine Pause oder ein Nachdenken zu signalisieren. So lautete der Untertitel: Punkt, Punkt, Punkt Lehrstück Punkt, Punkt, Punkt Theater Punkt, Punkt, Punkt Pädagogik Punkt, Punkt, Punkt. Statt der die Begriffe trennenden Punkte hätte man auch drei Gedankenstriche setzen können; denn es sollte und musste damals - noch - darüber nachgedacht werden, wie diese drei Begriffe wohl zusammenpassen oder ob sie überhaupt zusammengehören, ob sie sich stören, ausschließen oder aber ergänzen. Wir, das heißt diejenigen, die damals die Zeitschrift herausgaben - es waren das hauptsächlich Florian Vaßen, in dessen Verlag die 
damals noch kleine Publikation (etwa 300 Exemplare betrug die Auflage; jetzt sind es fünfmal so viele) erschien, und ich - wir wollten auf jeden Fall einen fruchtbaren, konstruktiven und nachdenklichen Widerspruch der drei Begriffe anregen. Wir gingen vom Gelingen einer Art Dialektik aus: Die Begriffe sollten sich durchaus stören, ergänzen und zusammen etwas Neues anregen - etwas, was unserer Meinung nach in der westdeutschen Bundesrepublik Deutschland zu der Zeit noch nicht vorhanden war.

Auch konnte man alle drei Begriffe in einem Atemzug lesen und wie ein Wort hören: Lehrstücktheaterpädagogik! Oder man koppelte nur zwei Begriffe aneinander. Dann konnte man diese Wortzusammensetzungen bilden: Lehrstückpädagogik - Theaterpädagogik - Lehrstücktheater - auch vielleicht: pädagogisches Theater. So ähnlich konnte man kreativ mit den drei Begriffen „Lehrstück“, „Theater“ und „Pädagogik“ umgehen.

Ich komme später noch einmal speziell auf den möglicherweise ungewohnten Begriff „Lehrstück“ zurück.

\section{Korrespondenzen}

Nun aber etwas zum Haupttitel der Zeitschrift: „Korrespondenzen“. Er sollte die Leseanweisung, die Dynamik (oder Dialektik) der drei Begriffe anregen. Die Begriffe und die dahinter stehenden Arbeitsformen und Tätigkeitsfelder und kommunikativen Muster sollten miteinander korrespondieren (lernen), miteinander in Verbindung treten. Und auch die Menschen, die kreativ, theatral, pädagogisch dort jeweils tätig sind, sollten miteinander in Korrespondenz, also in Austausch treten. Dafür hatten wir im Übrigen in der Tat auch die Zeitschrift gegründet. Wir wussten von vielen Personen, die schon lange oder neuerdings erst auf den Feldern von Lehrstück, Theater und/oder Pädagogik - mit je unterschiedlichem Schwerpunkt - tätig waren. Diese Kolleginnen und Kollegen hatten unterschiedliche Berufe oder befanden sich noch in der Ausbildung oder im Studium. Manche waren Lehrer/Lehrerinnen, Erwachsenenbildner, Erzieher, Religionspädagogen, Gewerkschafter, Sozialpädagogen, Literaturwissenschaftler, Schauspieler, Regisseure usw. Und man arbeitete oft ohne Kontakt $\mathrm{zu}$ anderen. Es fehlte an kollegialem Austausch und an einer gemeinsamen Fachsprache. Und Austausch, Korrespondenz sind ja gerade dann wichtig, wenn man an etwas Neuem arbeitet: Man braucht dafür Stützungen, Supervision, Anregungen, Hilfen, Fachgespräche, Ergänzungen und Kritik durch andere. Der Stückeschreiber Bertolt Brecht nannte so etwas eine „Produktionsberatung“.

Nochmals gesagt: Eine Fach-Zeitschrift bietet sich dafür an! Und wir können auch sagen: In den Anfängen der Zeitschrift hatten wir es nicht immer leicht, genügende Artikel zu bekommen. Das hat sich sehr geändert: Die Praktiker, die die Zeitschrift lesen, schreiben auch in ihr, indem sie über Praxis schriftlich reflektieren. Und die Theoretiker haben ein Forum, um Theorien im Feld von Wissenschaft und Praxis vorzustellen und um sich durch Praxis und Diskurs 
zu präzisieren in ihren Forschungen. Nicht immer ist es leicht, Theoretiker von Praktikern streng zu trennen - und das ist innerhalb eines praxeologisch fundierten Arbeitsansatzes, wie ihn Pädagogik und Theater darstellen, auch gut so! Die Zeitschrift ist eine Institution der Weiterbildung geworden!

Aber: Im Felde von Theaterpädagogik bedarf es auch der sehr direkten, unmittelbaren Begegnung mit Kolleginnen und Kollegen, des persönlichen und reflektierenden Zusammenspiels, der S elbstbildung, d er Selbstqualifizierung, des persönlich-körperlichen Agierens. Das kann etwa in Kurzzeit-workshops oder mittels einer Spielwoche zur Selbstverständigung geschehen. Und so geschah es damals auch zusätzlich zur Publizistik.

Neben der Zeitschrift und mit ihr korrespondierend fanden die spielpraktischen Exerzitien, die theaterpädagogischen Experimente statt. Aus solchen Versuchen wurden neue theaterpädagogische Konzepte entwickelt. Dort wurden theoretische Konzepte praktisch erprobt. Sie wurden dadurch überprüft. Es entstanden aus kollektiver Arbeit neue Gedanken und man stärkte sich untereinander. Es entstanden wissenschaftliche Diplom- und Magister- und Doktorarbeiten sowie Buchpublikationen, die das Neue fixierten.

Für die Zeitschrift bedeutete es, dass sie 1994 umbenannt werden konnte: Die bei der Gründung noch lose beieinander stehenden und zusätzlich durch drei Punkte voneinander getrennten Begriffe „Lehrstück“, „Theater“ und „Pädagogik“ fielen weg, und die Zeitschrift hieß fortan zwar weiter „Korrespondenzen“, aber mit einem neuen Untertitel, nämlich: „Zeitschrift für Theaterpädagogik“. Die erste Ausgabe mit dem Untertitel „Zeitschrift für Theaterpädagogik“ war ein Dreifach-Heft, das sich gerade mit dem Begriff, der nun wegfiel, befasste: Der Heft-Schwerpunkt umfasste fast 130 Seiten zu Brechts Lehrstücken!

\section{Zeitschrift für Theaterpädagogik}

Der neue, eindeutige Untertitel zeigt nun schon deutlich an, dass man es nicht mehr additiv mit drei Begriffen zu tun hat, sondern der jetzt verwendete Begriff „Theaterpädagogik“ signalisierte, dass etwas Neues in die Pädagogik und ins Theater kam: die Theaterpädagogik als eine integrative Disziplin!

Der Haupttitel „Korrespondenzen“ hatte sich wie eine trade mark, wie ein label eingeführt, so dass wir erst 2005 - also 20 Jahre nach Begründung der Zeitschrift - uns trauten, den neuen Untertitel „Zeitschrift für Theaterpädagogik“ zum Haupttitel zu machen - und das Wort „Korrespondenzen“ rutschte zur Erinnerung an die Anfänge in den Untertitel. Wir hatten nach langen Jahren des Experimentierens und der Selbstqualifikation jetzt d en Mut, mit solchen wissenschaftlichen Fachpublikationen zu ,konkurrieren', die eine ähnliche Titel-Fassung für ihr jeweiliges, etabliertes Fachgebiet gewählt hatten, nämlich etwa die „Zeitschrift für Pädagogik“, „Kölner Zeitschrift für Soziologie und Sozialpsychologie“, „Zeitschrift für Erziehungswissenschaft“, „Zeitschrift für Soziologie“. Und wir kooperieren mit Zeitschriften, die ähnlich arbeiten, z. B. mit den Internet-Publikationen „Scenario“ oder „SpielArt“. 


\section{Lehrstück - learning play}

Bei der sukzessiven Neufassung des Titels fiel e in B egriff w eg - s ehr zum Bedauern mancher Kolleginnen und Kollegen der ersten Stunde - nämlich der Begriff „Lehrstück“. Und dabei stand gerade dieser Theater- und BildungsAnsatz Pate bei der Gründung der Zeitschrift 1985. Ich werde deshalb jetzt einiges zum Lehrstück sagen - gewissermaßen eine Danksagung dafür, dass wir dieser neuen Form des Theater-Machens begegnet sind. Personell gebührt der Dank dem Stückeschreiber Bertolt Brecht, der diesen Typus, dieses Theaterformat praktisch-theoretisch begründet hat. Und ein weiterer Dank gebührt dem Forscher Reiner Steinweg, einem Literaturwissenschaftler und späteren Sozialwissenschaftler sowie Friedens-und Konfliktforscher, der in den 1970er Jahren Brechts Lehrstücke wiederentdeckt und dessen Theorie systematisch entfaltet hat in seiner Untersuchung mit dem Titel „Das Lehrstück. Brechts Theorie einer politisch-ästhetischen Bildung“ (Stuttgart 1972, 2. verbessere Auflage 1976). Reiner Steinweg stand als Person und als Forscher wie Florian Vaßen und ich im Kreise derjenigen Korrespondenten, aus denen die spätere Zeitschrift für Theaterpädagogik entstand.

Was nun ist ein Lehrstück? Bertolt Brecht wählte für eine englische Publikation diese Übersetzung: ,learning play“. In den 1920er/1930er Jahren gab er in "Zur Theorie des Lehrstücks" diese prägnante Beschreibung dessen, was ein Lehrstück nach seinem Verständnis sein soll: "Das Lehrstück lehrt dadurch, daß es gespielt wird, nicht dadurch, daß es gesehen wird. Prinzipiell ist für das Lehrstück kein Zuschauer nötig, jedoch kann er natürlich verwertet werden. Es liegt dem Lehrstück die Erwartung zugrunde, daß der Spielende durch die Durchführung bestimmter Handlungsweisen, Einnahme bestimmter Haltungen, Wiedergabe bestimmter Reden und so weiter gesellschaftlich beeinflußt werden kann. Die Nachahmung hochqualifizierter Muster spielt d abei eine große Rolle, ebenso die Kritik, die an solchen Mustern durch ein überlegtes Andersspielen ausgeübt wird. (...) Es braucht sich keineswegs um die Wiedergabe gesellschaftlich positiv zu bewertender Handlungen und Haltungen zu handeln; auch von der (möglichst großartigen) Wiedergabe asozialer Handlungen und Haltungen kann erzieherische Haltung erwartet werden (...) Die Form der Lehrstücke ist streng, jedoch nur damit Teile eigener Erfindung u nd a ktueller Art desto leichter eingefügt werden können" (Bertolt Brecht 1967: 1024). Bertolt Brecht äußerte sich am Beispiel seines umstrittenen Lehrstücks "Die Maßnahme": "'Dieses Stück ist nicht zum Lesen gemacht. Dieses Stück ist nicht zum Ansehen gemacht'. 'Wozu denn?'. 'Zum Spielen. Zum Spielen unter sich"' (Bertolt Brecht nach Steinweg 1972: 261); es sollen Selbstverständigungsprozesse zwischen den Experimentell-Spielenden und mit der Stückvorlage und unter Berücksichtigung der Welt, in der man sich befindet, eingeleitet werden. Dieser theaterpädagogische Ansatz erinnert an das sozialpsychologisch begründete Gruppen-Lernmodell von Ruth Cohn - an ihre „Themenzentrierte Interaktion“ (im Deutschen abgekürzt: TZI), die das Ich, das Wir, das Thema und den globe, also die Welt, in eine gleichberechtigte Kommunikations-Dynamik bringen 
will. Wir können im Anschluß an Brechts Lehrstück-Vorschläge sein GruppenLernmodell also „Theaterzentrierte Interaktion“ nennen (und im Deutschen auch die Abkürzung TZI verwenden).

Was war mit dem Lehrstückansatz von Bertolt Brecht für die Theaterpädagogik gewonnen? Vier Punkte will ich nennen:

- Ein literarisch sehr versierter Autor, ein Dichter, ein Dramatiker stellte (s)ein künstlerisches, ästhetisches Werk der Pädagogik, der Erziehung, der Bildung zur Verfügung.

- Ein Künstler verstand sich als sozialer, politischer, künstlerischer und kritischer Pädagoge.

- Mit Hilfe von learning plays konnten interessierte Gruppen von Menschen sich selbstverständigen, sich stärken (heutigerFachbegriff: empowerment), gesellschaftlich als $N G O$ öffentlich aktiv werden und mit politisch-sozialen Themen emanzipatorisch und zivil-gesellschaftlich experimentieren, um einen demokratischen Experimentalismus zu etablieren - in Anlehnung an den pragmatistischen Philosophen und Pädagogen John Dewey formuliert („Kunst als Erfahrung“ war der Titel eines seiner Bücher).

- Man kann sich durch Lehrstückspiel im künstlerisch-kommunikativen Medium selbst ernst nehmen und wertschätzen (Fachterminus: self esteem). Ohne das Wort schon zu benutzen, hatte Bertolt Brecht (s)ein Konzept von Theaterpädagogik entwickelt.

Das war damals in den 1970er Jahren für viele eine Eröffnung und eine Neuerung innerhalb der Szene von Spiel- und Theaterpädagogik in der Bundesrepublik Deutschland. Vorher hatte man primär aus dem Spielbegriff, aus dem Verständnis von Spielen als anthropologischer und entwicklungspsychologischer Größe, auch aus dem kindlichen Spiel und aus dem reformpädagogischen Schulspiel heraus Theaterpädagogikals Spielpädagogik, als sozial-ästhetisches Rollenspiel, als Darstellendes Spiel in der Schule verstanden.

Es muß angemerkt werden, dass Bertolt Brecht - auch - teilweise in dieser Tradition stand - siehe dazu seine Übersetzung ins Englische: learning play, also SPIEL, und siehe auch Brechts Zusammenarbeit mit seinem späteren Verleger Peter Suhrkamp, der aus der deutschen Reformpädagogik kam. Und die sozialistische Arbeiterbildung war Brecht natürlich auch bekannt. Jedoch verschiebt er die Begründung der Pädagogik (seiner Pädagogik) von der Anthropologie oder Entwicklungspsychologie auf die Soziologie als Wissenschaft von der Gesellschaft und ihrer Ökonomie und als Wissenschaft vom vergesellschafteten Menschen. Eingreifendes Denken war für Brecht ein in Gesellschaft praktisch eingreifendes Denken. Also eingegriffen werden sollte in die Lebenswirklichkeit von Menschen. Brecht wollte eine „Lebenskunst“, die er als die höchste aller Künste verstand, entwickeln. Wenn das kein pädagogisches Ziel ist! 


\subsection{EXKURS I: Transformationen des Alltags (Ute Pinkert)}

Ute Pinkert, seit 2006 Professorin am Institut für Theaterpädagogik der Universität der Künste Berlin, macht in ihrer Untersuchung zu „Transformationen des Alltags" vertraut mit „Theaterprojekte(n) der Berliner Lehrstückpraxis und Live Art bei Forced Entertainment“. Ihre Untersuchungen belegen die Wichtigkeit der Lehrstück-Arbeit für die Entwicklung eines neuen Verständnisses von Theaterpädagogik bzw. für die „Lernform Theater“:

„Die Berliner Lehrstückpraxis steht im Zusammenhang einer spezifischen Art theaterpädagogischer Projektarbeit, die von Hans Martin Ritter Ende der 1970er Jahre unter dem Begriff der ,Lernform Theater' zusammengefasst wurde. Diese versteht sich als ein Arbeitszusammenhang, in dem, Verfahrensweisen, Arbeitsformen und Projektmodelle entwickelt und untersucht (wurden), die soziale und ästhetische Momente zu vermitteln suchen: die Abbildung, Rekonstruktion und Untersuchung subjektiver Erfahrungszusammenhänge und der dort eingebundenen Momente kulturellen und ästhetischen Verhaltens und ihre Ausformung und Entfaltung durch Theaterarbeit.' [Ritter 1981c: 131 (das ist: Hans Martin Ritter: Theater als Lernform. Beiträge zur Theorie und Praxis Pädagogischer Theaterverfahren. Studienmaterialien Spiel- und Theaterpädagogik. Berlin 1981, Anm. gk)]“. (Pinkert 2005: 142) - Interessant nun ist, dass und wie Ute Pinkert einen Sprung in heutiges theatrales ErfahrungenMachen, in eine andere ,Lernform Theater' unter den Bedingungen einer aktuellen Postmoderne macht - mit Bezug auf Lehrstück-Praxis.

Sie befragt Protagonisten der „Theaterprojekte der Berliner Lehrstückpraxis“ der 1970er Jahre (Hansjörg ,Scotch' Maier, Jörg Richard, Hans Martin Ritter) im Kontext eines postmodernen Weltaneignungsansatzes, wie sie ihn aktuell bei der „britischen Performancegruppe ,Forced Entertainment"“ findet (Kapitel I II.2, 213 ff.): „Im B ezug a uf d ie Berliner Lehrstückpraxis als eines Beispiels reflexiv a usgerichteten ästhetischen Lernens wäre ... zu konstatieren, dass innerhalb des Theaterspielens bei Forced Entertainment nicht die Erkenntnis eigener Verhaltensmuster (bzw. innerer Modelle) mittels deren Verkörperung und spezifischer Deutung ... im Mittelpunkt steht, sondern das Erfahren von Konstruktionsmöglichkeiten des eigenen Selbst, wie es über die (embodied) Ausführung verschiedener Konstruktionen ermöglicht wird.“ (2005: 316)

„Dabei kann vorausgesetzt werden, dass die Aneignungsprozesse performativen Wissens, welche innerhalb einer performativ-pragmatischen Ästhetik wie bei Forced Entertainment vor sich gehen, in anderer Weise beschrieben werden müssen als in der Berliner Lehrstückpraxis. Entsprechend der ... Veränderung von Auffassungen zu ,Wahrnehmung', ,Körper' und ,Bedeutungserzeugung' wäre damit auch der Begriff des Lernens im Rahmen eines noch auszuarbeitenden ,Konzepts der Verkörperung' . . . neu zu überdenken ....". (2005: 316)

„Im Unterschied zur Berliner Lehrstückpraxis, die auf die bewusste Veränderung der ,inneren Modelle' und damit letztlich auf deren Angleichung an bestimmte (durch die Spielleitenden vertretene) Werte zielte, wird bei Forced Entertainment die Differenz der Perspektiven und 
Handlungsweisen der Spielenden zum entscheidenden - ästhetisch wie politisch begründeten - Wert. Die Präsentation von (unzähligen) Varianten der Umsetzung einer Spielregel, wie sie das Prinzip der ,offenen Wiederholung' ... möglich macht, verbindet sich dabei mit dem Anspruch, die Wirklichkeit auf der Bühne im wertschätzenden Zusammenspiel verschiedener, differierender Menschen und Materialien zu konstruieren und zu präsentieren. In dieser Arbeitsweise, die auf die Etablierung von Beziehungen ausgerichtet ist, welche so wenig wie möglich funktionalisiert, verwertungs- und machtorientiert sind, sieht die Gruppe den entscheidenden politischen Ansatz ihrer Arbeit ... In diesem Sinne kann der konsequenzverminderte Raum des Theaters hier auch als Ort beschrieben werden, an dem in durchaus, utopisch' zu nennender Weise Beziehungen oder Haltungen im Sinne von Möglichkeiten der ,WeltBegegnung' handelnd erprobt werden. In diesem miteinander handelnden und ,ausgehandelten' Entwurf von Beziehungen (,first-hand') liegt, so die These (von Ute Pinkert, Anm. d. Verf.), das entscheidende ethischästhetische Potential von Theaterspielen in der postmodernen Gegenwart." (2005: 320 ff.)

Unseres Erachtens beschreibt Ute Pinkert hier etwas, was im neueren Spiel mit Lehrstücken auch zu beobachten ist: Die Spiel-Dynamik, die Gruppendynamik, das Probieren und Verwerfen von Varianten ist durchaus spiel-methodisch im Lehrstück-Spiel vertreten - auch wenn eine meta-theoretische Vergewisserung beim Lehrstück Differenzen zu manchen postmoderne Konzepten aufweist. Eine kritische Postmoderne bzw. eine postmoderne linke Theoriebildung, die sich dialektisch auch bei Marx vergewissert und wie sie namentlich in Bezug auf Brechts Werk von Terry Eagleton (Die Illusionen der Postmoderne) und Fredric Jameson (Lust und Schrecken der unaufhörlichen Verwandlung aller Dinge) betrieben wird, kann auf der phänomenologischen Ebene mit postmodernen Beschreibungen einig gehen. Durch den experimentellen Ansatz, der dem Lehrstück-Spiel eigen ist, berührt es sich in übender Praxis (vgl. Vaßen: Lernen und Üben) durchaus mit avancierter Postmodernität (wenn die sich einer experimentellen Dialekt der Moderne zugehörig weiß).

\section{Theaterarbeit in sozialen Feldern}

Bis zum Jahre 1993, bis zum Doppel-Heft Nr. 17/18, trug die Zeitschrift das Wort „Lehrstück“ im Untertitel. Manche Ideen von Bertolt Brecht sind in seiner Nachfolge variiert worden und wirkten auf die Konzeptentwicklung von Theaterpädagogik(en). Ich weise auf zwei besonders hin:

- Ende der 1970er Jahre erschien in deutscher Übersetzung das Buch des Brasilianers Augusto Boal mit dem Titel „Theater der Unterdrückten“. Es hatte weltweit großen Erfolg - und hat es bis heute. Augusto Boal kam unter anderem auch von Brecht her, aber er betonte viel stärker die soziale Empirie von unmittelbarer Unterdrückung und der Verunmöglichung gesellschaftlicher Partizipation und auch den Ausschluss der Wahrnehmung von Menschenrechten. Er strebte mittels seines Theaterkonzeptes 
eine umfassende Alphabetisierung (ganz direkt und metaphorisch gesprochen) und kulturelle Dynamisierung an - damit Unterdrückung minimiert werden kann. Theater konnte so ein Modell und eine Methode gesellschaftlicher Beteiligung werden. Augusto Boal entfaltete sein theaterpädagogisches Programm bis hin zu tiefenpsychologisch beeinflussten Arbeitsweisen und er arbeitete auch an einer Ästhetik des Theaters der Unterdrückten. [Heft 34 (1999) der „Korrespondenzen“/Zeitschrift für Theaterpädagogik widmet sich den theaterpädagogischen Ideen und Praktiken von Augusto Boal.]

- Am Beginn des 21. Jahrhunderts wurde eine EU-weite theaterpädagogische Aktivität, ausgehend von Kolleginnen und Kollegen aus Österreich, ins Leben gerufen, unter anderem in Kooperation mit dem University College Cork: „Theaterarbeit in sozialen Feldern“ hieß dieses Programm. Dazu liegt ein spezielles Curriculum vor (siehe das Buch von Koch/Roth/Vaßen/Wrentschur). Es bündelte die vielen verschiedenen, sich sozial verpflichtet fühlenden theaterpädagogischen Ansätze, etwa das Zielgruppentheater, das community theatre, das theatre for development, das eben erwähnte Theater der Unterdrückten. Eigentlich müsste in diesem Zusammenhang noch an etwas Drittes erinnert werden. Denn auch die traditionellen Theater verstanden sich zunehmend mehr als gesellschaftlich-künstlerische Lern- und Erfahrungsorte und entwickelten theaterpädagogische Programme für ihr junges und älteres Publikum (etwa Jugendclubs an Theatern - geleitet von Theaterpädagogen).

Das zu tun aber war nicht allein eine Folge der außer-theatralen oder theaterpädagogischen Aktivitäten. Nein, es lag auch daran, dass sich das Theaterverständnis selbst erweitert hatte. Man denke etwa an performances, an das sog. postdramatische Theater (nach Hans-Thies Lehmann), an Publikumsbeteiligung und Mitspieltheater und an die Mischung der Genres: Film, Bild, Musik, Objekt- und Materialtheater, Circus, Varieté, Aufnahme von einheimischen Volkstheaterformen ins künstlerische Theater, Übernahmen von Theatertraditionen anderer Länder und Kulturen, Theaterexpeditionen, Theater als Recherche und als theateranthropologische Forschung und Darstellung, als Dekonstruktion, als Mischung von sog. Professionellen und sog. Amateuren. Das Format feature wurde im Dokumentar-Drama verwendet (z. B. postmaster@theater-daktylus.de), das Erzähltheater wird erinnert und angewandt [vgl. Ulrike Hentschel/Gundel Mattenklott (Hrsg.): Erzählen. Narrative Spuren in den Künsten; Reiner Steinweg (Hrsg.) in Zusammenarbeit mit Gerd Koch: Erzählen, was ich nicht weiß - und den 2011 beginnenden Studiengang an der Berliner Universität der Künste: Künstlerisches Erzählen - Storytelling in Art and Education (Broschuere_Storytelling_zIW.pdf)]. Auch das autobiografische Theater zeigt Proben lebensgeschichtlichen Werdens auf der Bühne vorgenommen usw. Theater zeigt seine Arbeitsprozesse und seine eigenen Zweifel nun häufig öffentlich und gibt ein Muster für öffentliche Erkenntnisweisen - damit dem pädagogischen Tun nicht unähnlich ... ! 
Mit anderen Worten und zusammengefasst: Theaterpädagogische Formen und Herangehensweisen berührten sich mit neuen Formen und Herangehensweisen des Theaters als Institution - und: Institutionelle Theater zeigten ihre Produktionsweisen theater-öffentlich, veränderten sie und verstanden sich immer mehr als eine komplexe und widersprüchliche Einheit von Bühne und Zuschauerraum. Nicht nur die Schauspielkunst wurde entwickelt; auch die Zuschaukunst wurde mit-entwickelt - was übrigens eine alte Forderung von Bertolt Brecht war. Und damit sind wir wieder bei einem weiten Verständnis von Theaterpädagogik! Wenn Theater und Pädagogik sich intensiv begegnen, also miteinander korrespondieren, dann verändern sie sich - zu ihren Gunsten! Sie spielen sich dann nicht gegeneinander aus, sondern erweitern und ergänzen sich (heute kann man von einem Synergie-Effekt sprechen).

\subsection{EXKURS II: Theaterpädagogik als „Lebenswissenschaft“/“"life science"?}

Die Fähigkeit des Theaters, in verdichteter Form unterschiedliche Sprachen und verschiedenartige Logiken gleichzeitig zu Gehör zu bringen und miteinander zu verschränken, kann in ihrer Bedeutung für das individuelle wie für das kollektive Leben schwerlich überschätzt werden.

Theater ist das Ergebnis und das Erlebnis einer ebenso transgenerationellen wie transkulturellen Tätigkeit, die gewiß zu den komplexesten und kreativsten Aktivitäten gehört, die sich Menschen unterschiedlicher Herkunft bislang geschaffen haben. Kulturen und Gesellschaften entwickeln $\mathrm{zu}$ bestimmten Zeiten und innerhalb bestimmter Kontexte ein Zusammenlebenswissen, das sich nicht nur immer weiter anreichern, sondern auch in mehr oder minder starkem Maße verloren gehen kann. Eine am Lebenswissen der Theater der Welt ausgerichtete Theaterwissenschaft und -pädagogik vermag die Vielfalt der theatral geschaffenen polylogischen Strukturen nicht nur zu analysieren, sondern für eine individuelle wie gesellschaftliche Entwicklung fruchtbar zu machen, die von möglichst komplexen, verschiedene Kulturen und Diskurse querenden Denkweisen geprägt ist. Ein derartiges Wissen ist für unsere Gesellschaften überlebensnotwendig.

Ich habe soeben eine Passage aus Prof. Dr. Ottmar Ette (2010): „Über Literaturwissenschaft als Lebenswissenschaft. Perspektiven einer anhebenden Debatte“ zitiert - mit kursiv gesetzten Veränderungen: Immer dort, wo bei Ette (Romanist an der Universität Potsdam) das Wort „Literatur“ (oder Varianten) steht, habe ich Theater (mit Varianten) geschrieben.

Ettes Gedanken - wie die durch ihn maß-geblich angestoßene Debatte ${ }^{1}$ sollten auch uns in der Theaterpädagogik anregen - vielleicht in folgender Weise [geschrieben von mir für die Bundesarbeitsgemeinschaft (BAG) Spiel und Theater aus Anlaß des Aktionstags: "Kultur gut stärken"-21. Mai 2011]:

\footnotetext{
${ }^{1}$ siehe Asholt/Ette (Hrsg) (2010) [weitere Informationen zur Veröffentlichung unterunter: http://www. uni-potsdam.de/romanistik/ette/lebenswissen.html - 5.6.2011]
} 
Warum das Kulturgut ,Spiel und Theater' gerade heute stärken? Weil beides ganz alte, globale menschliche Verhaltensweisen sind. Und weil sie ganz zeitgenössische Verhaltensweisen sind!

Die heutige Welt wird versucht, mit etwa solchen Begriffen systematisierend und konstruktiv zu beschreiben und zu begreifen: Multikulturalität, Transkulturalität, Interkulturalität, Weltinnenpolitik, Globalität/Mondialität, Multiversum, Kreolisierung (creolité), mobiles Weltbewusstsein/Weltwissen, Poly-/Pluri-Zentrizität, Kosmobilität, Differenzsensibilität, Migration/Wanderung als Weltphänomen ...

Theater und Spiel sind Verhaltensweisen, die ihren wesentlichen Grund genau in den eben notierten Stichworten haben. Zur Erinnerung: Wandernde Spielleute; die Fahrenden, Zigeuner (Tsiganes), die misstrauisch beäugt wurden; Trickspieler; Komödianten haben unsichere Existenzen; Leben mit Vorurteilen und Kriminalisierung (unsichere Kantonisten); es gibt Verbote gegenüber dem Fremden in Spiel und Theater, auch Zensur von Texten; zugleich selbstbewusste Übernahme von Themen und Methoden aus allen Richtungen der Welt; Zeigen und Verlebendigung des Normabweichenden; Überschreitung von Regeln; Genießen und Wollust; Theaterleute als vogelfreie Leute, Vaganten, Obdachlose, freie und couragierte Frauen, Überlebenskünstler ... Zusammengefasst: Transitorische Existenzen und Verhaltensweisen. Und: Das Unsichere ist (der zu sichernde Ort) von Spiel und Theater...

Also: Spiel und Theater $=$ eine entfaltete Tradition und Praxis des Unsicheren. Ein welthaltiges, immaterielles Kulturerbe; eines, was sich immer wieder neu erfindet. Und was immer aufs Neue die Welt bereichert. Dieses Kulturgut Spiel und Theater gilt es zu stärken. Und: Es stärkt Kultur(en) seinerseits. Es verfügt in diesen Hinsichten über einen umfangreichen Erfahrungsschatz.

Spiel und Theater sind Zeitgenossen der Weltverhältnisse, in denen wir leben und die wir als Subjekte in Gleichheit und Freiheit - auch und gerade - ästhetisch eingreifend gestalten wollen und können. ${ }^{2}$

\section{Theater und Schule}

Ich habe bisher fast gar nicht von Schule oder Schulunterricht oder Schultheater oder Darstellendem Spiel in der Schule oder theatre in education oder drama in education gesprochen (siehe aber den „Bundesverband Theater in Schulen (BV TS)“ bvts@live.de, www.bvts.org). Das war Absicht. Denn ich möchte weder Pädagogik noch Theater nur auf einen zwar wichtigen gesellschaftlichen Ort, nämlich die Schule, beziehen. Wenn wir etwa über Musik oder Sport reden, dann reduzieren wir das nicht auf schulischen Musikunterricht oder Sportunterricht. So sollte es auch mit dem Theater sein! Ich will aber kurz

\footnotetext{
${ }^{2}$ Prof. Dr. Gerd Koch, Vorstand BAG Spiel \& Theater, http://www.bag-online.de/aktuell/ kulturgutstaerken.html - 5.6.2011
} 
auf eine Entwicklung hinweisen, die in Berlin begann und sich dann in anderen Großstädten Deutschlands fortsetzte: „Theater und Schule“ (TuSch). Es geht dabei um die Kooperation von je einem Theater mit einer Schule über eine längere Zeit. Beide Institutionen schließen einen Kooperations-Vertrag und helfen sich: Theaterleute kommen als Experten in die Schule, in den Unterricht; Schüler gehen in das Theaterhaus und arbeiten unter theaterprofessionellen Gesichtspunkten dort - nicht nur, indem sie dort auf der Bühne spielen, sondern indem sie alle Gewerke, also die Produktionsform eines Theaterbetriebs als Ganzem kennen lernen. Die Theaterleute wiederum lernen durch die Kooperation mit der Schule die komplexe Bildungsinstitution Schule und deren besondere Erziehungsaufgaben kennen. Beide Einrichtungen nehmen sich in Gemeinsamkeiten und Unterschieden durch Realkontakt und nicht über Vorurteile/Stereotypen wahr. Zugleich bilden sich die Theaterleute wie die Schulleute (und das sind Schüler, Lehrer und auch Eltern) weiter durch praktisches Zusammenwirken - durch Korrespondenz. Gegenseitige Fremdheit wird abgebaut (es findet unter den jeweils professionell Arbeitenden ein job enrichment statt).

\section{Theaterpädagogik: Selbsthilfe und organisierte Ausbildung}

Die Ausbildung zu Theaterpädagogen ist in Deutschland sehr vielfältig und traditionell eine Domäne von freien Anbietern bzw. geschieht in der Form von Selbstorganisation, als Selbsthilfe (self help) derjenigen, die sich weiter qualifizieren wollen, da sie z. T. schon in Feldern der Theaterpädagogik tätig sind (man kann diesen Ansatz als peer education bezeichnen). Es gibt in den einzelnen deutschen Bundesländern „Landesarbeitsgemeinschaften (LAG) Spiel und Theater", die Fort- und Weiterbildungen zum Spielleiter oder - erweitert - zum Theaterpädagogen anbieten. Ein Spielleiter ist ein ,kleiner' Theaterpädagoge. Die katholische und evangelische Kirche haben traditionell Arbeitsgemeinschaften und Bildungseinrichtungen zur Qualifizierung v on Mitarbeitern für theaterpädagogische Aufgaben. Seit 1990 gibt es einen großen „Bundesverband Theaterpädagogik" (BuT), der nicht nur selbst ein Bildungsangebot macht, sondern er hat im Mai 1994 seine „Bildungskonzeption“ beschlossen, die notwendige Ausbildungsinhalte für Berufsbilder des Theaterpädagogen zusammenstellte.

Es können folgende Arbeitsbereiche für Theaterpädagogik benannt werden: Theater, Theater- und Musikhochschulen, Hochschulen, Einrichtungen der Theater-, Ausdrucks- und Spielforschung, Schulen, Kindergärten, Soziale Arbeit, medizinischer Bereich, Sport, Wirtschaft, theaterpädagogische Zentren. Über folgende Kompetenzen soll ein Theaterpädagoge verfügen: Darstellungskompetenz, Handlungskompetenz, Gleichgewichtskompetenz (verstanden als Gleichgewicht zwischen emotionalen darstellerischen und kognitiven Dimensionen), konstruktive Kompetenz, ethisch-kommunikative Kompetenz und 
ästhetische Kompetenz. 1999 hat der Bundesverband Theaterpädagogik eine Qualifizierungsoffensive g estartet, d ie R ahmenrichtlinien für die Ausbildung von Theaterpädagogen festlegte. So sollen Spielpädagogen eine GrundlagenAusbildung von etwa 600 Unterrichtsstunden bekommen und qualifizierte Theaterpädagogen müssen weitere 1100 Unterrichtsstunden belegen. Solche Ausbildung geschieht an freien Institutionen (private Unternehmen, gemeinnützige Vereine), die vom Bundesverband Theaterpädagogik eine Ausbildungslizenz bekommen, wenn sie nachweisen können, dass sie über genügend qualifiziertes $P$ ersonal $v$ erfügen, $\mathrm{u} m$ e ine $\mathrm{d}$ auerhafte, $\mathrm{m}$ eist einbis zweijährige Ausbildung gewährleisten zu können. Zur Ausbildung gehört das Absolvieren eines theaterpädagogischen Eigenprojekts, und die Abschlussprüfung wird unter Teilnahme eines vom Bundesverband ausgewählten Fachgutachters abgenommen. Die Absolventen bekommen ein Zertifikat mit dem Titel „Theaterpädagoge“ mit dem Zusatz „BuT“. Seit Juni 2007 ist dieser Titel markenrechtlich, das heißt „, als eingetragene Marke“, geschützt. Diese Ausbildungsstätten für Theaterpädagogik müssen übrigens in regelmäßigen Abständen nachweisen, dass sie in der Lage sind, ihre Ausbildung qualitätsvoll zu gestalten. Außerdem hat der Bundesverband Theaterpädagogik eine Kommission gebildet, die Anträge von bisher schon theaterpädagogisch arbeitenden Menschen daraufhin prüft, ob deren praktische und langdauernde Erfahrung es geboten erscheinen lässt, dass sie die Berufsbezeichnung „Theaterpädagoge BuT" führen dürfen.

Die „Bundesarbeitsgemeinschaft Spiel und Theater“, kurz „BAG Spiel und Theater" genannt, ist die älteste westdeutsche Vereinigung auf dem Gebiete der Förderung von Spiel und Theater. Sie hat 1994 Prof. Dr. Ulrike Hentschel (Universität der Künste Berlin) und mir den Auftrag gegeben, ein „Kern-Curriculum“ für die Ausbildung in Theaterpädagogik zu erstellen. Dieses Ausbildungsmuster benennt folgende Lernfelder und Gegenstände, die mindestens erfüllt werden müssen, um eine umfassende Ausbildung zu gewährleisten: Theaterpraxis, Theatertheorie, Medialität und Subjektivität, Technik und Organisation. Fünf Kompetenzen sollen bei den Theaterpädagogen ausgebildet werden: Subjekt-Kompetenz, künstlerische Kompetenz, theoretische Kompetenz, Vermittlungskompetenz (also methodisch-didaktische Kompetenz) und technische/handwerkliche/organisatorische Kompetenz. Dieses Kern-Curriculum wurde Vorbild für akademische und außerakademische Ausbildungsgänge, auch in der Lehrer-Aus- und -Weiterbildung.

Seit 1995 gibt es in Deutschland eine „Ständige Konferenz Spiel und Theater an deutschen Hochschulen". Das ist eine Interessensvertretung von Personen, die in der akademischen Ausbildung für Theaterpädagogik tätig sind. Einige Daten zu akademischen Ausbildungen: Das am weitesten fortgeschrittene StudienModell für Lehrer, die das Fach Theater bzw. Darstellendes Spiel in der Oberstufe des Gymnasiums professionell unterrichten, wurde von fünf Hochschulen und Universitäten (in den norddeutschen Städten Braunschweig, Hannover und Hildesheim) als Gemeinschaftsprojekt entwickelt. Hier können die Studenten das Fach wie ein anderes, traditionelles Unterrichtsfach im Lehramtsstudium 
studieren. Das ist neu für das bundesdeutsche Schulwesen. Die Hochschule der Künste Berlin, jetzt heißt sie Universität der Künste (UdK), bietet Theaterpädagogik mit dem Abschluss Master als postgradualen Studiengang an. Dieser Studienabschluss qualifiziert nicht für den staatlichen Lehrerberuf. Die UdK hat aber eine lange Tradition in der berufsbegleitenden Weiterbildung von Lehrern in Spiel- und Theaterpädagogik. An dieser Universität sind verschiedene Doktorarbeiten zu theaterpädagogischen Themen entstanden.

Die Fachhochschule Osnabrück bildet an ihrem „Institut für Theaterpädago-

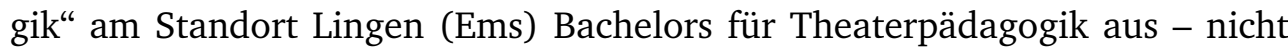
mit der Befähigung für das Lehramt an staatlichen Schulen - wohl aber für die Arbeit an Theatern, in der Erwachsenen- und Jugendbildung oder in der innerbetrieblichen Ausbildung bei Unternehmen. Die Hochschule für Musik und Theater in Rostock bildet im Kontext der Schauspielausbildung auch in der Theaterpädagogik aus. Sehr fortgeschritten und pädagogisch wie theaternah ist die deutschsprachige Ausbildung in Zürich in der Schweiz. An vielen Hochschulen (ehemals Fachhochschulen/Universities for Applied Sciences) - meistens solchen für Soziale Arbeit - gibt es kulturpädagogische Ausbildungssegmente, die auch Theaterpädagogik umfassen. Das Ausbildungsspektrum reicht konzeptionell von mehr ästhetischen/künstlerischen bis hin zu eher sozial-methodischen und -therapeutischen Verständnissen von Theaterpädagogik. Kolleginnen und Kollegen, die in solchen Arbeitsfeldern an Fachhochschulen tätig sind, haben 2003 einen Interessentenverband gegründet: den „Bundesarbeitskreis Kultur, Ästhetik, Medien (BAKÄM)“.

\section{Wörterbuch der Theaterpädagogik}

Im Arbeitszusammenhang der Alice-Salomon-Fachhochschule Berlin, in der ich tätig war, und mit fachlicher Unterstützung durch die Gesellschaft für Theaterpädagogik (die Florian Vaßen und ich leiten) sowie durch staatliche finanzielle Unterstützung haben Prof. Dr. Marianne Streisand und ich 2003 das erste deutschsprachige „Wörterbuch der Theaterpädagogik“ herausgegeben. Das Wörterbuch umfasst 299 Stichworte, die von 140 Experten aus dem In- und Ausland verfasst wurden. Dass erst 2003 das erste Wörterbuch der Theaterpädagogik herauskam, zeigt an, dass das Fach Theaterpädagogik verhältnismäßig jung ist. Aber ein Wörterbuch oder Lexikon bedeutet auch: Eine Disziplin zeigt selbstbewusst an, dass sie da ist und selbstbewusst am wissenschaftlich-fachlichen Diskurs teilnimmt!

Seit kurzem gibt es eine Forschung, die die vergleichsweise junge Geschichte der Theaterpädagogik unter dem Titel „Archäologie der Theaterpädagogik“ erforscht. Diese Arbeit wird von Prof. Dr. Marianne Streisand betrieben. Sie leitet auch das Deutsche Archiv der Theaterpädagogik [vgl. Deutsches Archiv für Theaterpädagogik (DATP) (Hrsg.):10 Jahre Theaterpädagogik-Studium in Lingen]. 


\section{Evaluation}

Zu den Aufgaben der Theaterpädagogik gehört meines Erachtens auch, ihre Arbeit zu evaluieren, um sie zu verbessern. Das kann ganz unmittelbar und kleinformatig im Anschluss an Kurse, workshops oder Unterricht geschehen, und es sollte neben statistischen Daten auch und gerade sinnliche, emotionale Bewertungseindrücke sammeln. Das wusste im übrigens schon Bertolt Brecht, der zu solchem Zweck 1930 einen Fragebogen - leider nur für Besucher - zum politischen Lehrwert seines Lehrstück „Die Maßnahme“ entwickelte. Wichtig sind spiel-prozess-begleitende Beschreibungen, Vergewisserungen und mehrperspektivische Analysen, sog. Wirksamkeitsdialoge, die integraler Bestandteil einer partizipativen Didaktik sein müssen. Folgende Methoden werden angewandt und können empfohlen werden: Teilnehmende Beobachtung, beschreibende Teilnahme, Eigenprotokolle, Arbeitstagebücher, Kreatives Schreiben und Anfertigung von Zeichnungen zur theaterpädagogischen Reflexion, Briefaustausch unter den theaterpädagogisch Lehrenden und Lernenden, Protokoll-Schreiben und gemeinsames Auswerten, Aufführungen der theaterpädagogischen Kritik zur Verfügung stellen, Film-Foto-Video-Dokumentationen erstellen - letzteres auch, um Unterrichts- und Lehrfilme zu haben.

Grundsätzliche, wissenschaftliche Forschungsmodelle sind in den letzten Jahren publiziert worden - beispielsweise:

Antonios Lenakakis (er ist seit 2010 Professor an der Aristoteles Universität Thessaloniki) hat Lehrer in Deutschland befragt, wie sich ihre theaterpädagogische Ausbildung generell auf ihre Berufsrolle als Lehrer ausgewirkt habe (es ging nicht um das Schulfach Theater). Es zeigte sich, dass theaterpädagogische Methoden die Professionalität in allen Unterrichtsfächern verbessert und dass Kommunikationsfähigkeit gestärkt und entfaltet wird.

Romi Domkowsky hat 2010 eine sozialwissenschaftliche, qualitative und quantitative empirische Untersuchung von Darstellendem Spiel in der Schule mit dem Titel „Theaterspielen - und seine Wirkung“ abgeschlossen. Sie beforscht zugleich eine Vergleichs-Schülergruppe, die keine theaterpädagogische Ausbildung bekommt. Die Untersuchung wird in absehbarer Zeit veröffentlicht werden. Die Forscherin hat bereits an der Alice-Salomon-Hochschule Berlin und an der UdK Berlin Untersuchungen über die längerfristige Wirkung von Theaterpädagogik bei Kindern und Jugendlichen vorgelegt (Biographie-Forschung wurde etwa mit narrativen Interviews mit quantitativen Methoden kombiniert).

2008 lieferte Ute Pinkert im von ihr herausgegebenen Band mit dem Titel „Körper im Spiel“. „Wege zur Erforschung theaterpädagogischer Praxen“.

2007 erschien eine empirische Untersuchung mit dem Titel „Potentiale Ästhetischer Praxis in der Sozialen Arbeit. Eine Untersuchung zum Bereich Kultur - Ästhetik - Medien in Lehre und Praxis“" (herausgegeben von Petra Marquardt und Wolfgang Krieger). Man kann diese Untersuchung als ein SelbsthilfeForschungs-Projekt bezeichnen; denn ausgehend von vorhandenen Problemen um die Anerkennung der Fächer/Bereiche Ästhetik, Kultur und/oder Medien in der Ausbildung (speziell an Fachhochschulen vornehmlich im Feld der Sozialen 
Arbeit) wurde eine umfangreiche Untersuchung, die „Konturen und Profil Ästhetischer Bildung in der Sozialen Arbeit" zeigen soll, herausgearbeitet. Die Untersuchung von Marquardt und Krieger hat eine flächendeckende Befragung zum methodischen und konzeptionellen Selbstverständnis von akademischen Lehrern unternommen und dieses ergänzt durch stichprobenhafte Befragung von Absolventinnen der Ausbildung. Das Forschungsmuster kann spezialisiert für die Theaterpädagogik genutzt werden.

In Westdeutschland ist seit über 50 Jahren der wichtigste internationale theaterpädagogische Anreger und Koordinator die „BAG Spiel und Theater" (auch der Bund Deutscher Amateurtheater ist international aktiv und baut neuerdings seine theaterpädagogische Mitglieder-Fortbildung aus).

Ganz und gar nicht unwichtig ist das Veranstalten von Festivals mit theaterpädagogischer Prägung, denn das sind Bildungs-, Weiterbildungs- und Evaluationsmöglichkeiten durch gegenseitigen Austausch neuer theaterpädagogischer Arbeitsweisen. Auch internationale Kontakte können hergestellt und gepflegt werden. Das war im übrigen auch eine Form, die in der damaligen ostdeutschen DDR gängig war.

Prof. Dr. Kristin Wardetzky hat am „Theater der Freundschaft" in Berlin (DDR) Untersuchungen zur Akzeptanz und Wirkung des Kinder- und Jugendtheaters durchgeführt.

Ute Pinkert nennt „drei Frauen, die die Theaterpädagogik der DDR maßgeblich geprägt“ haben: „Christel Hoffmann, Angelika Wiesner und Hedwig Golpon“" (Pinkert 2008: 127).

"(T)heaterpädagogische Arbeit in der DDR“ ging „,von Künstlern aus“ und war „darauf gerichtet ..., nicht professionell Schauspielende mit der Theaterkunst vertraut zu machen (ebda, 8). Mit Prof. Dr. Christel Hoffmann war man international - etwa durch die ASSITEJ - verbunden und man pflegte eine umfangreiche theaterpädagogische Arbeit mit Kindern und mit Arbeitern in Betrieben. Festivals dienten dem gegenseitigen Austausch und der pädagogischen Steuerung durch Preisverleihungen.

Meines Erachtens müssen gerade in der Theaterpädagogik Festivals sein: Unser Fach benötigt den lebendigen Austausch. Wir kennen Buchmessen, um Literatur-Produkte zu zeigen; wir kennen Kunstausstellungen, um Kunst und Künstler wahrzunehmen. Und wir benötigen etwa Kinder- und Jugend- und Senioren-Theater-Festivals, um unsere Produkte und Prozesse vorzustellen und zu kritisieren. Und wir brauchen sie, um theaterpädagogisch zu lernen. Und wir brauchen sie auch, um uns gesellschaftlich und kulturell wie politisch zu positionieren. Es gibt in der Tat viele überregionale und regionale und institutionen- oder adressatenspezifische Festivals dieser Art in Deutschland. 


\section{Theaterpädagogik - eine gesellschaftliche und kulturelle Aufgabe}

Theaterpädagogik probt und arbeitet einerseits geschützt und konzentriert, gewissermaßen intim in verschiedenen Lern- und Erfahrungsorten

UND

Theaterpädagogik geht in die Öffentlichkeit - ja, sie stellt Öffentlichkeit mittels ästhetisch-pädagogischer Eingriffe her

UND

riskiert aktives Leben als kulturelle, ästhetische Bildung

UND

vitalisiert mit ästhetisch-pädagogischen Mitteln soziale und Gruppenverhältnisse und Lebensentwürfe - ist lebensbegleitendes Lehren und Lernen.

UND

Diese komplexe Aufgabenvielfalt muss in der Bundesrepublik Deutschland immer wieder publizistisch, politisch und wissenschaftlich in Erinnerung gebracht und erkämpft werden.

Literaturhinweise

Asholt, Wolfgang/Ette, Ottmar (Hrsg.) (2010): Literaturwissenschaft als Lebenswissenschaft. Tübingen: Narr

Belgrad, Jürgen (1997): TheaterSpiel. Ästhetik des Schul- und Amateurtheaters. Hohengehren: Schneider

Boal, Augusto (1989): Theater der Unterdrückten. Frankfurt am Main: Suhrkamp

Brecht, Bertolt (1967): Gesammelte Werke, Bd. 17. Frankfurt am Main: Suhrkamp

Brunkhorst, Hauke (Hrsg.) (1998): Demokratischer Experimentalismus. Politik in der komplexen Gesellschaft. Frankfurt am Main: Suhrkamp

Bülow-Schramm, Margret/Gipser, Dietlinde/Krohn, Doris (Hrsg.) (2007): Bühne frei für Forschungstheater. Oldenburg: Paulo Freire

Bundesverband Theaterpädagogik: Bildungskonzeption. In: Korrespondenzen. Zeitschrift für Theaterpädagogik, H. 19/20/21.

Cronin, Bernadette/Roth, Sieglinde/Wrentschur, Michael (Hrsg.) unter Mitarbeit von Gerd Koch und Florian Vaßen (2005): Training Manual for Theatre Work in Social Fields. Frankfurt am Main: Brandes \& Apsel

Deutsches Archiv der Theaterpädagogik (Hrsg.) (2009):10 Jahre TheaterpädagogikStudium in Lingen. Lingen: Eigenverlag 
Domkowsky, Romi (2008): Erkundungen über langfristige Wirkungen des Theaterspielens. Eine qualitative Untersuchung. Auf Spurensuche. Saarbrücken: Dr. Müller

Dörger, Dagmar/Nickel, Hans-Wolfgang (Hrsg.) (2005): Spiel- und Theaterpädagogik studieren. Uckerland: Schibri

Eagleton, Terry (1997): Die Illusionen der Postmoderne. Stuttgart, Weimar: Metzler

Hentschel, Ulrike (1996, 2. Aufl. 2010): Theaterspielen als ästhetische Bildung. Uckerland: Schibri

Hentschel, Ulrike/Koch, Gerd (1995): Kerncurriculum Theaterpädagogik. In: Korrespondenzen. Zeitschrift für Theaterpädagogik 23, 24, 25

Hentschel, Ulrike/Mattenklott, Gundel (Hrsg.) (2009): Erzählen. Narrative Spuren in den Künsten. Uckerland: Schibri

Hilliger, Dorothea (2006): Theaterpädagogische Inszenierung. Uckerland: Schibri

Hoffmann, Christel/Schneider, Wolfgang (Hrsg.) (2006): spiel.raum.theater. Frankfurt am Main: Peter Lang

Hoffmann, Klaus/Handwerg, Ute/Krause, Katja (Hrsg.) (2007): Theater über Leben. Uckerland: Schibri

Jameson, Fredric (1999): Lust und Schrecken der unaufhörlichen Verwandlung aller Dinge. Hamburg: Argument

Koch, Gerd (1988, zuerst 1979): Lernen mit Bert Brecht. Frankfurt am Main: Brandes \& Apsel

Koch, Gerd (1995): Theatralisierung von Lehr-Lernprozessen. Milow: Schibri

Koch, Gerd/Steinweg, Reiner/Vaßen, Florian (1983): Asoziales Theater. Spielversuche mit Lehrstücken und Anstiftung zur Praxis. Köln: Prometh

Koch, Gerd/Naumann, Gabriela/Vaßen, Florian (1999. 2. Auflage 2011): Ohne Körper geht nichts. Lernen in neuen Kontexten. Milow: Schibri

Koch, Gerd/Roth, Sieglinde/Vaßen, Florian/Wrentschur, Michael (Hrsg.) (2004): Theater in sozialen Feldern/Theatre Work in Social Fields. Frankfurt am Main: Brandes \& Apsel

Koch, Gerd/Streisand, Marianne (Hrsg.) (2003): Wörterbuch der Theaterpädagogik. Uckerland: Schibri 
Lehmann, Hans-Thies (1999): Postdramatisches Theater. Frankfurt am Main: Verlag der Autoren

Lenakakis, Antonios (2003): Paedagogus Ludens. Uckerland: Schibri

Marquard, Petra/Krieger, Wolfgang (Hrsg.) (2007): Potentiale Ästhetischer Praxis in der Sozialen Arbeit. Hohengehren: Schneider

Pinkert, Ute (2005): Transformationen des Alltags. Theaterprojekte der Berliner Lehrstückpraxis und Live Art bei Forced Entertainment. Modelle, Konzepte und Verfahren kultureller Bildung. Berlin. Uckerland: Schibri

Pinkert, Ute (2008): Körper im Spiel. Wege zur Erforschung theaterpädagogischer Praxen. Uckerland: Schibri

Ritter, Hans Martin (1981): Theater als Lernform. Beiträge zur Theorie und Praxis pädagogischer Theaterverfahren. Studienmaterialien Spiel- und Theaterpädagogik. Berlin: Eigenverlag

Ritter, Hans-Martin (2009): Zwischenräume: Theater - Sprache - Musik. Grenzgänge zwischen Kunst und Wissenschaft. Uckerland: Schibri

Steinweg, Reiner (1972, 2. verbesserte Auflage 1976): Das Lehrstück. Stuttgart: Metzler

Steinweg, Reiner/Wolfgang Heidefuß/Peter Petsch (1986): Weil wir ohne Waffen sind. Ein theaterpädagogisches Forschungsprojekt zur Politischen Bildung. Nach einem Vorschlag von Bertolt Brecht. Frankfurt am Main: Brandes \& Apsel

Steinweg, Reiner (1995, 2. Aufl. 2005): Lehrstück und episches Theater. Frankfurt am Main: Brandes \& Apsel

Steinweg, Reiner (Hrsg.) in Zusammenarbeit mit Gerd Koch (2006): Erzählen, was ich nicht weiß. Die Lust zu Fabulieren und wie sie die politische, soziale und therapeutische Arbeit bereichert. Uckerland: Schibri

Streisand, Marianne/Hentschel, Ulrike/Poppe, Andreas/Ruping, Bernd (Hrsg.) (2005): Generationen im Gespräch. Archäologie der Theaterpädagogik I. Uckerland: Schibri

Vaßen, Florian (2010, 2. Aufl.): Lernen und Üben. Erfahrung und Wahrnehmung, ,Unstetigkeit' und ,Einsehen' im ästhetisch-sozialen Prozess des Theater-Spielens. In: Ders. (Hrsg.): Korrespondenzen. Theater - Ästhetik - Pädagogik. Uckerland: Schibri, 129 - 146

Wildt, Beatrix/Hentschel, Ingrid/Wildt, Johannes (Hrsg.) unter Mitarbeit von Gerd Koch (2008): Theater in der Lehre. Wien, Berlin: Lit

Zeitschrift für Theaterpädagogik (neu: ISSN 1865-0756) - erscheint im SCHIBRI Verlag

$<$ www.schibri.de $>$ 